\title{
Learning Kinematic Models for Articulated Objects
}

\author{
Jürgen Sturm ${ }^{1}$, Cyrill Stachniss ${ }^{1}$, Vijay Pradeep ${ }^{2}$, Christian Plagemann ${ }^{3}$, \\ Kurt Konolige $^{2}$, Wolfram Burgard ${ }^{1}$ \\ ${ }^{1}$ Univ. of Freiburg, Dept. of Computer Science, D-79110 Freiburg, Germany \\ ${ }^{2}$ Willow Garage, Inc., 68 Willow Road, Menlo Park, CA 94025 \\ ${ }^{3}$ Stanford University, CS Dept., 353 Serra Mall, Stanford, CA 94305-9010
}

\begin{abstract}
Topic: estimation, prediction
Oral presentation or poster presentation
\end{abstract}

Home environments are envisioned as one of the key application areas for service robots. Robots operating in such environments are typically faced with a variety objects they have to deal with or to manipulate to fulfill a given task. Many objects are not rigid since they have moving parts such as drawers or doors. Understanding the spatial movements of parts of such objects is essential for service robots to allow them to plan relevant actions such as door-opening trajectories. Ideally, robots are able to autonomously infer these articulation models by observation. In this work, we therefore investigate the problem of learning kinematic models of articulated objects from observations. As an illustrating example, consider the left three images of Figure 1 which depict two examples for observations of the door of a microwave oven and a learned, one-dimensional description of the door motion.

Our problem can be formulated as follows: Given a sequence of rigid body poses from observed objects parts, learn a compact kinematic model describing the whole articulated object. This kinematic model has to define (i) which parts are connected, (ii) the dimensionality of the latent (not observed) actuation space of the object, and (iii) a kinematic function between different body parts in a generative way allowing a robot to reason also about unseen configurations. Our approach is related to recent work of Katz et al. [1] who learn planar kinematic models for articulated objects such as scissors by manipulating the object as well as to the work of Yan and Pollefeys [4] who present an approach for learning the structure of an articulated object from feature trajectories under affine projections.

The contribution of this work is a novel approach for learning actuation models based on observations only. Our method is able to robustly detect the connectivity of the rigid parts of the object and to estimate accurate articulation models from a candidate set. Our approach allows for selecting the best model among parametric, expert-designed transformation templates (rotational and prismatic models), and nonparametric transformations that are learned from scratch requiring minimal prior assumptions. To obtain a parameter-free description, we apply Gaussian processes [2] as a non-parametric regression technique to learn flexible and accurate models. To find the low-dimensional description of the moving parts, we furthermore apply local linear embedding [3], which is a non-linear dimensionality reduction technique.

We implemented our approach on a real robot and tested it by estimating models of different objects, including a door of a microwave oven (see Figure 1 left), a cabinet with drawers (see Figure 1 right), a garage door (see Figure 2), and a table moved on the ground plane (see Figure 3). Our technique allows to learn accurate models for different articulated objects. We regard this as an important step towards autonomous robots understanding and actively handling objects in their environment. 

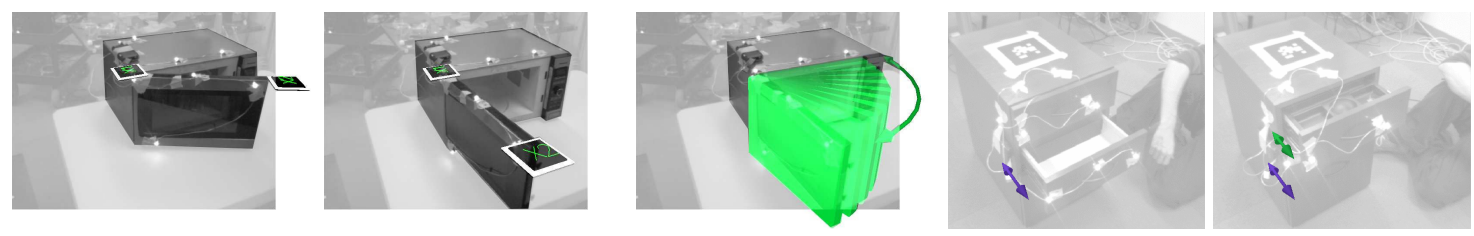

Figure 1: Left: examples for observations of a moving door of a microwave oven and an illustration of the learned 1-dimensional kinematic model. Right: Illustrations of the latent action variables (using the arrows) for a cabinet with two drawers during learning.
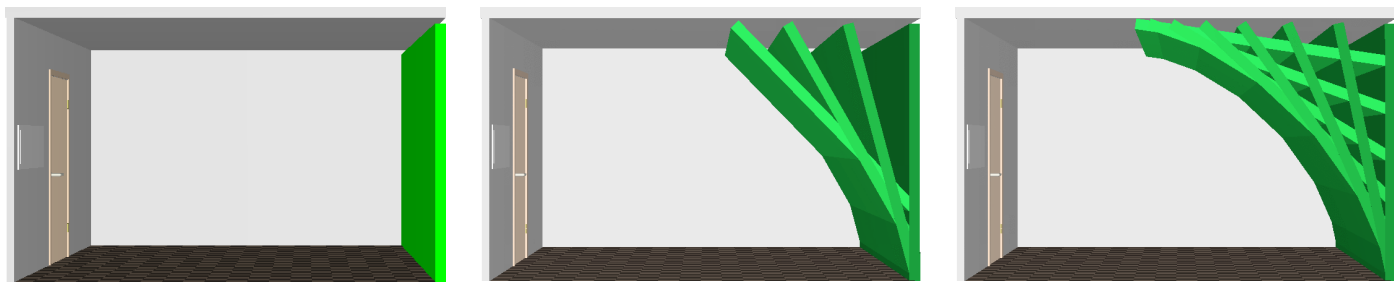

Figure 2: Illustration of the motion of a garage door predicted by our non-parametric model. The door runs in a horizontal rail on the ceiling and in a vertical rail on the right. This results in a movement than cannot be described by a prismatic or rotational model but using our non-parametric model. From left to right: Illustration of the model while integrating new observations.
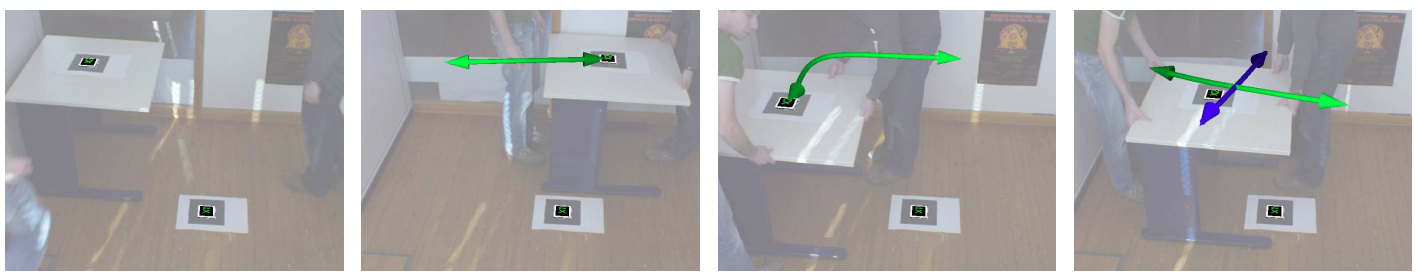

Figure 3: Four steps while learning a model of a table that is moved on the ground plane. The arrows indicate the latent action variable of the currently selected model. Finally, our non-parametric model with 2 degree of freedom explains the movements best.

\section{References}

[1] D. Katz, Y. Pyuro, and O. Brock. Learning to manipulate articulated objects in unstructured environments using a grounded relational representation. In Robotics: Science and Systems, 2008.

[2] C.E. Rasmussen and C.K.I. Williams. Gaussian Processes for Machine Learning. The MIT Press, Cambridge, MA, 2006.

[3] S.T. Roweis and L.K. Saul. Nonlinear dimensionality reduction by locally linear embedding. Science, 290(5500):2323-2326, 2000.

[4] J. Yan and M. Pollefeys. Automatic kinematic chain building from feature trajectories of articulated objects. In CVPR, 2006. 